\title{
Efecto de la escarificación y estratificación sobre la germinación in vitro de Aristotelia chilensis (Molina) Stuntz
}

\section{Effect of scarification and stratification on the in vitro germination of Aristotelia chilensis (Molina) Stuntz}

\author{
Mario Rodríguez Beraud ${ }^{1 *}$, Jocelyne Tampe Pérez ${ }^{2}$, Nelson Hormazábal Vásquez ${ }^{1}$, Ximena Araneda \\ Durán ${ }^{1}$, Ricardo Tighe Neira ${ }^{1} \&$ Paz Cárcamo-Fincheira ${ }^{1}$
}

${ }^{1}$ Escuela de Agronomía, Facultad de Recursos Naturales, Universidad Católica de Temuco, Casilla 15-D, Temuco, Chile. ${ }^{2}$ Unidad de Tecnología y Procesos, Departamento de Ingeniería Química, Universidad de La Frontera, Francisco Salazar 01145, Temuco, Chile.

*marodrig@uct.cl

\begin{abstract}
RESUMEN
Con el fin de mejorar la germinación de semillas de Aristotelia chilensis, se establecieron dos ensayos in vitro. En el primero, se realizó escarificación y se comparó con un testigo. En el segundo, se aplicó estratificación fría por diferentes períodos de tiempo $\left(2,4,6\right.$ y 8 semanas a $4{ }^{\circ} \mathrm{C}$ en oscuridad), más un testigo sin estratificar. Las semillas fueron germinadas en placas con agua y agar (AA) e incubadas por 60 días en cámara de cultivo a $25 \pm 2{ }^{\circ} \mathrm{C}$, con tubos fluorescentes de luz fría blanca bajo un fotoperíodo de $16 \mathrm{~h}$ luz, $8 \mathrm{~h}$ de oscuridad y una intensidad lumínica de $50 \mu \mathrm{mol} \mathrm{m} \mathrm{m}^{-2} \mathrm{~s}^{-1}$. En todos los tratamientos se logró valores promedios de germinación superiores a los testigos. La mayor germinación se obtuvo con escarificación $\left(\mathrm{G}_{\text {máx }}, 92 \%\right)$, no obstante la mayor velocidad inicial de germinación se registró con la estratificación por ocho semanas, alcanzando el menor tiempo medio de germinación (TM, 18 días). Los resultados demuestran que la semilla de A. chilensis presenta dormancia primaria de nivel moderado tanto exógena como endógena.
\end{abstract}

Palabras clave: Dormancia, maqui, propagación, semillas.

\begin{abstract}
Two in vitro assays were performing in order to improve Aristotelia chilensis germination rate. Scarification was tested in the first assay and compare to a control. In the second assay, cold stratification was applied during 2, 4, 6 and 8 weeks at $4{ }^{\circ} \mathrm{C}$ in darkness, plus an unstratified control. Seeds were germinated on Petri dish containing water and agar (WA), and incubated for 60 days in a controlled atmosphere at $25 \pm 2{ }^{\circ} \mathrm{C}$ using cold white fluorescent light tubes $\left(50 \mu \mathrm{mol} \mathrm{m}^{-2} \mathrm{~s}^{-1}\right.$ light intensity), under a photoperiod of $16 \mathrm{~h}$ light and $8 \mathrm{~h}$ of darkness. All treatments achieved higher average germination rates than the control. The highest germination rate was obtained with scarification $\left(\mathrm{G}_{\text {máx }}, 92 \%\right)$ though the fastest growth was achieved with 8 weeks of stratification, reaching the lowest average germination time of 18 days. The results demonstrate that $A$. chilensis seeds present a moderate level of primary dormancy, both exogenous and endogenous.
\end{abstract}

KEYwords: Dormancy, maqui, propagation, seeds.

\section{INTRODUCCIÓN}

Aristotelia chilensis (Molina) Stuntz (Elaeocarpaceae), también conocido como maqui, berrie-negro, maquei o queldrón, es un árbol, nativo de Sudamérica que crece en los bosques templados lluviosos del Sur de Chile y Argentina, que alcanza entre los 4 a $5 \mathrm{~m}$ de altura, y su fruto es una baya color violeta-negruzco de 5 a $6 \mathrm{~mm}$ de diámetro (Moesbach 1992, Hoffmann et al. 1992). Los frutos del maqui son considerados como uno de los berries más saludables de la naturaleza (Genskowsky et al. 2015), son ricos en polifenoles, taninos y antocianinas, tales como la delfinidina y cianidina, los cuales le otorgan una elevada capacidad antioxidante y antibacterial (Ruiz et al. 2010, Rubilar et al. 2011, Tanaka et al. 2013, Rodríguez et al. 2016, Romanucci et al. 2016, Genskowsky et al. 2016). Por tal razón, el maqui surge como un interesante recurso para la elaboración de extractos alimenticios y nutracéuticos (Alonso 2012, Brauch et al. 2015).

Para la propagación de $A$. chilensis se utiliza tanto la 
vía sexual como vegetativa. La propagación vegetativa se realiza a través de esquejes de tallos con la aplicación de hormonas de enraizamiento, principalmente ácido indol butírico (AIB) (Joublan et al. 1999, Palma 2001). En el caso de la propagación sexual, se recomienda la inmersión de las semillas en agua fría durante 48-72 h para obtener una buena germinación (Misle et al. 2011). No existen antecedentes sobre la germinación in vitro, solo hay estudios bajo condiciones naturales (Figueroa 2003) o con papel filtro en cámaras de germinación (Molina 2001), en cualquier caso los resultados muestran una baja germinación, entre 34 y 63\% (Vogel et al. 2005), lo que supone la existencia de mecanismos de dormancia presentes en la semilla.

La dormición o latencia puede definirse como el bloqueo que tiene lugar en una semilla viable y que le impide completar la germinación en condiciones favorables, dependiendo tanto de las características físiológicas como morfológicas de la semilla (Bewley et al. 2013, Baskin \& Baskin 2014). La dormancia primaria está dada por factores exógenos y endógenos. La dormancia exógena hace referencia a las condiciones ambientales básicas que determinan el proceso de germinación como disponibilidad de agua, luz y temperatura. En tanto, la dormancia endógena es inherente a las características internas de la semilla, tales como dormancia por embriones rudimentarios, inhibición metabólica u osmótica. La dormancia combinada (exógenaendógena) hace referencia a las combinaciones de latencia de la cubierta o el pericarpio con latencia fisiológica endógena (Baskin \& Baskin 2014). Para romper esta condición existen diversos mecanismos, tales como la escarificación y la estratificación. La escarificación es una técnica donde, por métodos mecánicos o químicos, se induce la germinación a través de la rotura, abrasión o ablandamiento de la cubierta seminal, haciéndola más permeable al movimiento del agua y gases (Azcón \& Talón 2008). Esta técnica ha sido aplicada con éxito en semillas de Fragaria $\mathrm{x}$ ananassa Duch. (Miller et al. 1992) y Empetrum hermaphroditum L. (Baskin et al. 2002). Por otra parte, la estratificación es un método de tratamiento donde las semillas húmedas son sometidas a un período de enfriamiento para una postmaduración del embrión (Hartmann \& Kester 2002). La estratificación ha mejorado la germinación de muchas especies, tal es el caso de Morus nigra L. (Koyuncu 2005), Uniola paniculata L. (Burgess et al. 2002) y Bunium persicum B. Fedtsch. (Sharifi \& Pouresmael 2006).

En especies nativas es frecuente observar una fuerte dormancia en sus semillas. Dicha condición les permite sobrevivir y eludir las condiciones ambientales desfavorables, a diferencia de las especies cultivadas cuyos mecanismos de dormancia han sido atenuados por efecto de la selección artificial y domesticación, facilitando su manejo productivo (Bewley et al. 2013).

La germinación in vitro permite obtener híbridos interespecíficos (Pereira et al. 2006) y somáticos (Correia
\& Canhoto 2010), inducción a la poliploidía (Dhooghe et al. 2011) y proporcionar las condiciones óptimas para la germinación de semillas provenientes de cruzamientos controlados para programas de mejoramiento vegetal, donde la semilla puede ser un material muy valioso y escaso (Rodríguez 2011, Rodríguez et al. 2014). También, se ha indicado que la germinación in vitro permite obtener una germinación más uniforme e incrementar el porcentaje y la tasa de germinación de las semillas (>95\%) (Bhattacharya $\&$ Khuspe 2000). El objetivo de este estudio fue incrementar la germinación de Aristotelia chilensis mediante las técnicas de escarificación y estratificación en condiciones in vitro, basándose en la hipótesis de que es posible reducir la dormancia por medio de la aplicación de estas técnicas.

\section{MATERIALES Y MÉTODOS}

OBTENCIÓN DE SEMILLAS

Las semillas se obtuvieron de frutos de maqui cosechados en diciembre de 2010, en la Ciudad de Rancagua, Región del Libertador Bernardo O'Higgins, zona central, 34 ${ }^{\circ} 10^{\prime} \mathrm{S}$; $70^{\circ} 45^{\prime} \mathrm{O}$. Los frutos se dejaron en maceración por 5 días para separar las semillas del fruto y posteriormente fueron secados en cámara a $25 \pm 2{ }^{\circ} \mathrm{C}$ por $24 \mathrm{~h}$. Las semillas secas fueron almacenadas en oscuridad a $4^{\circ} \mathrm{C}$ por tres meses antes de la siembra.

DESINFECCIÓN DE SEMILLAS

Las semillas fueron sumergidas en tres soluciones desinfectantes. La primera desinfección se realizó en una solución que contenía Mancozeb + Benomilo $\left(2 \mathrm{~g} \mathrm{~L}^{-1}\right.$ de Mancozeb, 0,6 $\mathrm{g} \mathrm{L}^{-1}$ de Benomil más unas gotas del tenso activo Tween 20) por 20 min sobre un agitador magnético. La segunda desinfección fue con una solución de alcohol $75^{\circ}$ por 5 segundos y por último las semillas fueron inmersas en hipoclorito de sodio $(\mathrm{NaOCl})$ al $1 \%$ por 10 min. Una vez concluida la desinfección se enjuagó tres veces con abundante agua destilada estéril. Todo el proceso de desinfección se realizó en una cámara de flujo laminar.

PRePARACIÓN DEL Medio de CUlTivo

Los medios de cultivo consistieron en agua destilada gelificada con $7 \mathrm{~g} \mathrm{~L}^{-1}$ de agar microbiológico ( ${ }^{\circledR}$ Merck, ajustando el pH a 5,8 con 1,0 N hidróxido de potasio (KOH) y 1,0 N de ácido clorhídrico ( $\mathrm{HCl})$, utilizando como buffer $3 \mathrm{mM} \mathrm{L}^{-1}$ de ácido 2-(N-morfolino) etanosulfónico (MES). Los medios fueron autoclavados por $20 \mathrm{~min}$ a $121^{\circ} \mathrm{C}$ a 1 atm de presión, para luego dosificar en cámara de flujo laminar $10 \mathrm{~mL}$ de medio por placas Petri de $60 \times 15 \mathrm{~mm}$.

\section{SiEMBRA Y CONDICIONES DE CULTIVO}

Se realizaron dos ensayos. En el primero se escarificó semillas desinfectadas, cortando con bisturí, parte de la 
testa en la zona opuesta a la cicatriz caruncular. Para el segundo, las semillas desinfectadas se sometieron a cuatro tratamientos de estratificación fría $(2,4,6$ y 8 semanas a 4 ${ }^{\circ} \mathrm{C}$ en oscuridad), más un testigo sin estratificar.

Para ello, un total de 25 semillas desinfectadas fueron sembradas por placa Petri con medio de cultivo en una cámara de flujo laminar, con un marco de siembra de 5x5 unidades. Al final de la siembra las placas fueron selladas con parafilm y luego incubadas en cámara de cultivo a 25 $\pm 2{ }^{\circ} \mathrm{C}$, con tubos fluorescentes de luz fría blanca bajo un fotoperíodo de $16 \mathrm{~h}$ luz, $8 \mathrm{~h}$ de oscuridad y una intensidad lumínica de $50 \mu \mathrm{mol} \mathrm{m}^{-2} \mathrm{~s}^{-1}$ (tubos Philips TLD 36W/54).

\section{VARIABLES EVALUADAS}

Se evaluó el porcentaje de germinación diariamente hasta los 60 días desde la siembra, germinación máxima $\mathrm{G}_{\text {máx }} \mathrm{y}$ tiempo medio de germinación (TM: $\mathrm{N}^{o}$ de días en que se alcanza la mitad de $\mathrm{G}_{\text {máx }}$ velocidad de germinación).

\section{ANÁlisis ESTADÍSTICO}

Se utilizó un diseño completamente al azar para todos los tratamientos. La unidad experimental correspondió a una placa con 25 semillas cada una. Cada tratamiento tuvo cuatro repeticiones. Se realizó análisis descriptivos para obtener la media y el error estándar con el software SPSS (versión 15.0 para Windows). Los resultados se expresan como promedio \pm el error estándar. Los gráficos fueron obtenidos utilizando el programa Sigma Plot versión 10.0.

\section{RESULTADOS}

ENSAYO 1: EFECTO DE LA ESCARIFICACIÓN SOBRE LA GERMINACIÓN IN VITRO DE $A$. CHILENSIS

La germinación máxima $\left(\mathrm{G}_{\operatorname{máx}}\right)$ de la semilla escarificada alcanzó un valor promedio mayor al testigo sin escarificar (92\% vs 64\%), iniciando su germinación 7 días antes que el control (Fig. 1). Después del inicio, la germinación se elevó en forma paulatina, describiendo una curva sigmoidea, que alcanza una mayor aceleración entre los 33 y 40 días en el caso de las semillas escarificadas y entre los 40 y 50 días en el testigo. El tiempo medio de germinación (TM), para semillas escarificadas fue de 21 días, mucho antes que los 42 días del testigo. A los 40 días se observó la mayor diferencia en la germinación entre ambos tratamientos (54\%), manteniéndose siempre la semilla escarificada con valores más elevados hasta el final del ensayo.

ENSAYO 2: EFECTO DE LA ESTRATIFICACIÓN SOBRE LA GERMINACIÓN IN VITRO DE SEMILLAS DE $A$. CHILENSIS

La germinación de las semillas de $A$. chilensis fue mejorada por el tiempo de estratificación. La mayor diferencia se observó entre el testigo y el tratamiento estratificado por 8 semanas, específicamente entre los 30 y 40 días.
No obstante, tanto al inicio como al final del período de evaluación de la germinación, no se observaron diferencias entre tratamientos. Las diferencias más evidentes entre tratamientos con 2, 4, 6, 8 semanas de estratificación y el control se observaron a los 40 días.

El TM para 2, 4, 6 y 8 semanas de estratificación se registró a los 22, 23, 21 y 18 días, respectivamente. Con el tratamiento de 8 semanas de estratificación se determinó un TM de 18 días, superando al control que obtuvo un TM de 43 días, logrando la mayor velocidad de germinación. En tanto que la $\mathrm{G}_{\text {máx }}$ para $2,4,6$, y 8 semanas de estratificación y el control fueron 66, 68, 70, 76 y $62 \%$ respectivamente (Fig. 2).

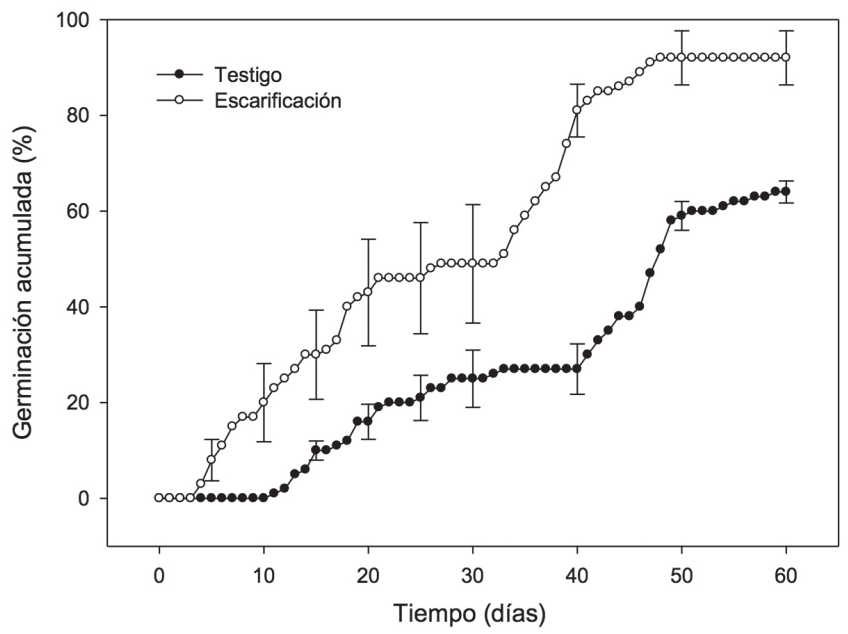

Figura 1. Efecto de la escarificación sobre la germinación acumulada de semillas de $A$. chilensis. Barras representan el error estándar. / Effect of scarification on accumulated seed germination of $A$. chilensis. Bars represent standard error.

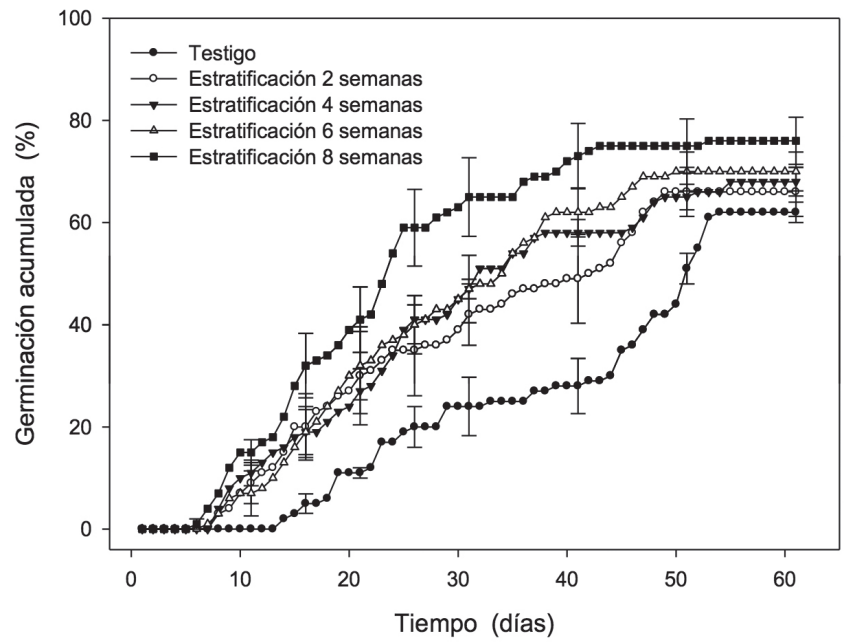

Figura 2. Efecto de la estratificación sobre la germinación de semillas de A. chilensis. Barras representan el error estándar. / Effect of stratification on seed germination of A. chilensis. Bars represent standard error. 


\section{DISCUSIÓN}

El porcentaje de germinación de la semilla escarificada mecánicamente fue superior a la semilla sin escarificar. Una situación similar ha sido reportada en Ugni molinae Turcz (Rodríguez 2011), Maihuenia patagonica (Phil.) Britton \& Rose (Masini et al. 2014), Parkia biglobosa (Jacq.) R. Br. ex G. Don (Aliero 2004), Prosopis ferox Griseb. (Ortega et al. 2002), Areca triandra Roxb. ex. Buch-Ham. (Yang et al. 2007), Mimosa foliolosa Benth. subsp. pachycarpa (Benth.) Barneby (Silveira \& Fernandes 2006), Astragalus filipes Torr. ex A. Gray y Dalea ornata (Douglas) Eaton \& Wright (Jones et al. 2016). La mayor germinación de la semilla escarificada mecánicamente puede explicarse, en semillas de endocarpio duro, por una mayor facilidad de expansión del embrión y/o por el aumento de la permeabilidad al agua y gases debido a la rotura de la cubierta seminal impermeable (Baskin \& Baskin 2014). Por otra parte, Faria et al. (2012) observaron que la escarificación mecánica y química fueron eficientes para romper la dormancia en condiciones in vitro de Senna macranthera (Dc. ex Collad.) H.S. Irwin \& Barneby tanto en condiciones de luz como en oscuridad, elevando la germinación de 18 a $80 \%$ con la escarificación mecánica.

Bajo condiciones naturales, la dispersión de semillas de A. chilensis ocurre de forma endozoocoria (Figueroa 2003). Los animales frugívoros contribuyen en la dinámica de dispersión biótica de las semillas, dada por la ingestión de los frutos. El paso de la semilla a través del tracto digestivo de especies frugívoras puede alterar la viabilidad, el porcentaje y velocidad de germinación, debido a las acciones mecánicas y químicas que ocurren en su interior (Figueroa 2003, Dennis et al. 2007, D'Avila et al. 2010, Gho-Illanes et al. 2015). Al respecto, Mora \& Soto-Gamboa (2011) observaron una mayor velocidad de germinación en semillas de Ugni molinae defecadas por el monito del monte (Dromiciops gliroides). Mancilla et al. (2013) describieron el efecto de la ingestión de semillas de Myrtus communnis L. por conejos europeos (Oryctolagus cuniculus) sobre la germinación, que se incrementó de 53,2 a $67,7 \%$, no afectando la viabilidad de la semilla. Similarmente, Carvalho et al. (2014) observaron el mismo efecto al comparar la germinación de semillas obtenidas desde frutos de Piper aduncum L. y Piper hispidinervum C. DC. y desde heces de murciélago (Carollia perspicillata). Entre los vertebrados frugívoros, las aves juegan un rol crucial en la dispersión de semillas, tal es el caso del zorzal patagónico (Turdus falcklandii) en Luma apiculata (DC.) Burret y Aextoxicon punctatum Ruiz et Pav. (Salvande et al. 2011), pudiendo mejorar los parámetros de germinación como ocurre con el tangara (Thraupis bonariensis) sobre el pimiento brasilero (Schinus terebinthifolius Raddi).

De acuerdo a lo observado en la Figura 2, las diferencias entre los tratamientos de estratificación versus el testigo se hicieron más evidentes a los 40 días con un leve aumento posterior, por lo cual no se aconseja un mayor tiempo de germinación, especialmente para la semilla de maqui estratificada por 8 semanas. En términos globales, estos resultados coinciden con la mayoría de las investigaciones reportadas para la estratificación en frío de diversas especies, tales como Anchusa crispa Viv., Centaurium maritimum (L.) Fritsch, Echium arenarium Guss., Glaucium flavum Crantz, Silene colorata L. (Benvenuti 2016). No obstante, Masini et al. (2014) no observaron diferencias significativas en la germinación al tratar semillas con estratificación húmeda fría en Maihueniopsis darwinii (Hensl.) F. Ritter var. hickenii (Britton \& Rose) R. Kiesling y Maihuenia patagonica. En otros casos la estratificación ha inhibido la germinación como lo reportado por Latsague et al. (2010) en Myrceugenia exsucca (DC.) O. Berg. Durante la estratificación en frío los niveles de ácido abscísico (ABA) decaen mientras que los contenidos de giberalinas aumentan (Hartmann \& Kester 2002). Al respecto, Chen et al. (2015) en semillas de Acer morrisonense Hayata observaron una tendencia al aumento de GAs $\left(\mathrm{GA}_{3}, \mathrm{GA}_{4}\right.$ y $\left.\mathrm{GA}_{7}\right)$ hasta las 8 semanas de estratificación fría y una disminución significativa en el contenido de ABA. En este mismo contexto, Darrudi et al. (2015) observaron en Rheum khorasanicum B. Baradaran \& A. Jafari un incremento de la germinación de 61 a $81 \%$ al combinar la estratificación $\left(2{ }^{\circ} \mathrm{C}\right.$ por 30 días $)$ con la adición de giberalinas $(48 \mathrm{~h})$. Por otra parte, este mismo autor señala un incremento de la germinación en relación a períodos más prolongados de estratificación y Brevis (2003) reporta un aumento en la velocidad de germinación en Eucryphia glutinosa (Poepp. et Endl.) Baill., como lo observado en esta investigación (Fig. 2). La estratificación cálida ha sido menos utilizada; investigadores como Dębska et al. (2013) han comparado estas técnicas en Malus domestica Borkh., resultando la estratificación fría superior a la cálida.

De acuerdo a los resultados obtenidos, se propone combinar ambas técnicas (escarificación y estratificación) para acelerar la germinación de A. chilensis, como lo realizado por Kaye \& Kuykendall (2001), quienes incrementaron la germinación en la semilla escarificada con lija de Lupinus sulphureus subsp. kincaidii de 45\% a 95\% al combinarla con estratificación a $4^{\circ} \mathrm{C}$, logrando un efecto sinérgico combinando ambas estrategias.

\section{CONCLUSIONES}

La semilla de $A$. chilensis presenta dormancia primaria de nivel moderado tanto exógena como endógena. En semillas no tratadas, la germinación in vitro puede alcanzar hasta un $60 \%$, porcentaje que se eleva cuando se aplican tratamientos de escarificación o estratificación. Para una mayor germinación final se recomienda la escarificación, no obstante con la estratificación fría por ocho semanas se 
obtiene una mayor velocidad inicial de germinación hasta los treinta días. Las técnicas utilizadas pueden ser empleadas para programas de mejoramiento y propagación masiva de esta especie.

\section{REFERENCIAS}

Aliero, B.L. 2004. Effects of sulphuric acid, mechanical scarification and wet heat treatments on germination of seeds of African locust bean tree, Parkia biglobosa. African Journal of Biotechnology 3(3): 179-181.

Alonso, J.R. 2012. Maqui (Aristotelia chilensis): Un nutracéutico chileno de relevancia medicinal. Revista de Farmacología de Chile 5(2): 95-100.

Azcón, J., TAlón, M. 2008. Fundamentos de fisiología vegetal. Segunda Edición. Interamericana-McGraw-Hill. Nueva York. 656 pp.

Baskin, C., Baskin, J. 2014. Seeds: ecology, biogeography, and evolution of dormancy and germination. Second edition. Elsevier, San Diego. 1573 pp.

Baskin, C., Zackrisson, O., Baskin, J.M. 2002. Role of warm stratification in promoting germination of seeds of Empetrum hermaphroditum (Empetraceae), a circumboreal species with a stony endocarp. American Journal of Botany 89(3): 486-493.

Benvenuti, S. 2016. Seed ecology of Mediterranean hind dune wildflowers. Ecological Engineering 91: 282-293.

Bewley, J.D., Bradford, K., Hilhorst, H., Nonogaki, H. 2013. Seeds: physiology of development, germination and dormancy. 3rd ed. Springer. New York. 392 pp.

Bhattacharya, J., Khuspe, S.S. 2000. In vitro and in vivo germination of papaya (Carica papaya L.) seeds. Scientia Horticulturae 91: 39-49.

Brauch, J.E., Buchweitz, M., Schweiggert, R.M., Carle, R. 2015. Detailed analyses of fresh and dried maqui (Aristotelia chilensis (Mol.) Stuntz) berries and juice. Food Chemistry 190(1): 308-316.

BRevis, P. 2003. Efecto de tratamiento pregerminativo sobre la germinación de semillas de Eucryphia glutinosa (Poepp. et Endl.) Baillon. Bosque 24(2): 79-84.

Burgess, T.L., Blazich, F.A., Nash, D.L. 2002. Seed germination of southern sea oats (Uniola paniculata) as influenced by stratification, temperature, and light. Journal of Environmental Horticulture 20(3): 180-183.

Carvalho, M.C., Uieda, W., Fonseca, R.C. B., Rossi, M.N. 2014. Frugivory and the effects of ingestion by bats on the seed germination of three pioneering plants. Acta Oecologica 55: 51-57.

Correia, S., Canhoto, J. 2010. Characterization of somatic embryo attached structures in Feijoa sellowiana Berg. (Myrtaceae). Protoplasma 242: 95-107.

Chen, S.Y., Chou, S.H., Tsai, C.C., Hsu, W.H., Baskin, C.C., Baskin, J.M., Chien, C.T., Kuo-Huang, L.L. 2015. Effects of moist cold stratification on germination, plant growth regulators, metabolites and embryo ultrastructure in seeds of Acer morrisonense (Sapindaceae). Plant Physiology and Biochemistry 94: 165-173.

Darrudi, R., Hassandokht, M.R., NAZER, V. 2015. Effects of moist stratification, $\mathrm{GA}_{3}$ and seed age on seed germination of Rheum khorasanicum B. Baradaran \& A. Jafari. Journal of Applied Research on Medicinal and Aromatic Plants 2: 168-173.

D’Avila, G., Gomes-Jr., A., Canary, A.C., Bugoni, L. 2010. The role of avian frugivores on germination and potential seed dispersal of the Brazilian Pepper Schinus terebinthifolius. Biota Neotropica 10(3): 45-51.

Dębska, K., Krasuska, U., Budnicka, K., Bogatek, R., GniazdowsKa, A. 2013. Dormancy removal of apple seeds by cold stratification is associated with fluctuation in $\mathrm{H}_{2} \mathrm{O}_{2}$, NO production and protein carbonylation level. Journal of Plant Physiology 170: 480-488.

Dennis A.J., Schupp, E.W., Green, R.J., Westcott, D.A. 2007. Seed dispersal. Theory and its application in a changing world. Oxford Shire, CAB International, United Kingdom. $684 \mathrm{pp}$.

Dhooghe, E., Van Laere, K., Eeckhaut, T., Leus, L., Van Huylenbroeck, J. 2011. Mitotic chromosome doubling of plant tissues in vitro. Plant Cell Tissue and Organ Culture 104: 359-373.

Faria, D.V., Lima, A.B.P., Barboza da Silva, N.C. 2012. Effects of scarification and nutrient mineral concentrations on the in vitro germination of Senna macranthera (Collad.) H. S. Irwin \& Barneby seeds. Emirates Journal of Food and Agriculture 24(4): 302-307.

FigueroA, J. 2003. Seed germination in temperate rain forest species of southern Chile: Chilling and gap. Dependency germination Plant Ecology 166: 227-240.

Genskowsky, E., Puente, L.A., Pérez-Álvarez, J.A., FernándezLópez, J., Muñoz, L.A., Viuda-Martos, M. 2015. Assessment of antibacterial and antioxidant properties of chitosan edible films incorporated with Maqui berry (Aristotelia chilensis), LWT- Food Science and Technology 64(2): 1057-1062.

Genskowsky, E., Puente, L.A., Pérez-Álvarez, J.A., FernándezLópez, J., Muñoz, L.A., ViudA-Martos, M. 2016. Determination of polyphenolic profile, antioxidant activity and antibacterial properties of maqui [Aristotelia chilensis (Molina) Stuntz] a Chilean blackberry. Journal of the Science of Food and Agriculture 96: 4235-4242.

Gho-Illanes, D., Smith-Ramírez, C., VÁsquez, I.A., Díaz, I. 2015. Frugivory of Persea lingue (Lauraceae) and its effect on seed germination in southern Chile. Gayana Botánica 72(2): 250-257.

Hartmann, H., Kester, D. 2002. Plant propagation. Principles and practices. Prentice Hall, New Jersey. 880 pp.

Hoffmann, A., Farga, C., Lastra, J., Veglozi, E. 1992. Plantas medicinales de uso común en Chile. Editorial Claudio Gay. Santiago, Chile. 273 pp.

Jones, T.A., Johnson, D.A., Bushman, B.S., Connors, K.J., SMITH, R.C. 2016. Seed dormancy mechanisms in basalt milkvetch and western prairie clover. Rangeland Ecology \& Management 69: 117-122.

Joublan, J.P., Berti, M., Serri, H., Wilckens, R., Poblete, P. 1999. Propagación vegetativa en maqui (Aristotelia chilensis). Agro-Ciencia 15(1): 33-38.

Kaye, T.N., Kuykendall, K. 2001. Effects of scarification and cold stratification on seed germination of Lupinus sulphureus ssp. kincaidii. Seed Science and Technology 29: 663-668. 
Koyuncu, F. 2005. Breaking seed dormancy in black mulberry (Morus nigra L.) by cold stratification and exogenous application of gibberellic acid. Acta Biologica Cracoviensia Series Botanica 47(2): 23-26.

Latsague, M., Sáez, P., Coronado, L. 2010. Tratamientos pregerminativos para Myrceugenia exsucca (Myrtaceae). Bosque 31(3): 243-246.

Mancilla, J.M., González-Redondo, P., Martín Vicente, A. 2013. Effects of rabbit gut passage on seed retrieval and germination of three shrub species. Basic and Applied Ecology 14: 585-592.

Masini, A.C.A., Rovere, A.E., PIRK, G.I. 2014. Requerimientos pregerminativos de Maihuenia patagonica y Maihueniopsis darwinii, cactáceas endémicas de Patagonia. Gayana Botánica (71)2: 188-198.

Miller, R., Scheereus, J., Erb, P. 1992. Enhanced strawberry seed germination through in vitro culture of cut achenes. Journal of the American Society for Horticultural Science (117)2: 313-316.

Misle, E., Garrido, E., Contardo, H., González, W. 2011. Maqui [Aristotelia chilensis (Mol.) Stuntz]-the amazing Chilean tree: A Review. Journal of Agricultural Science and Technology B 1: 473-482.

Moesbach, E. 1992. Botánica indígena de Chile. Andrés Bello, Santiago, Chile. 140 pp.

Molina, J.M. 2001. Preacondicionamiento de la semilla de maqui (Aristotelia chilensis) y descripción de sus cambios morfológicos en el proceso de germinación. Tesis Ingeniero Agrónomo. Facultad de Agronomía, Universidad de Concepción. Chillán, Chile. 32 pp.

Mora, J.P., Soto-Gamboa, M. 2011. Legítima dispersión de semillas Ugni molinae Turcz. (Myrtaceae), por monito del monte, Dromiciops gliroides. Gayana Botánica 68(2): 309312 .

Ortega, P., De Viana, M.L., Suhring, S. 2002. Germination in Prosopis ferox seeds: effects of mechanical, chemical and biological scarificators. Journal of Arid Environments 50: 185-189.

Palma, M. 2001. Propagación vegetativa de plantas femeninas de maqui Aristotelia chilensis (Mol.) Stuntz. Tesis Ingeniero Agrónomo. Facultad de Agronomía, Universidad de Concepción. Chillán, Chile. 27 pp.

Pereira, A.B., Carvalho-Oliveira, M., Ramírez, J., Galagovsky, L. 2006. Shooting control in Eucalyptus grandis $\mathrm{x}$ E. urophylla hybrid: comparative effects of 28homocastasterone and a 5a-monofluoro derivative. Plant Cell, Tissue and Organ Culture 86: 329-335.

Rodríguez, K., Ah-Hen, K.S., Vega-Gálvez, A., Vásquez, V., Quispe-Fuentes, I., Rojas, P., Lemus-Mondaca, R. 2016. Changes in bioactive components and antioxidant capacity of maqui, Aristotelia chilensis (Mol.) Stuntz, berries during drying. Food Science and Technology 65: 537-542.
Rodríguez, M. 2011. La propagation d'écotypes sélectionnés de murtilla (Ugni molinae Turcz.), une baie endémique du Chili: étude de la germination et stratégies de multiplication in vitro. Tesis doctoral. Institut des Sciences et Industries du Vivant et de l'Environnement, AgroParisTech, Ecole Doctorale ABIES, Paris, Francia. 232 pp.

Rodríguez, M., Chacón, M., Carrillo, R. 2014. Efecto de la concentración y de los componentes del medio de cultivo MS sobre la germinación in vitro de Ugni molinae. Bosque 35(1): 123-126.

Romanucci, V., D’Alonzo, D., Guaragna, A., Di Marino, C., Davinelli, S., Scapagnini, G., Di Fabio, G., Zarrelli, A. 2016. Bioactive compounds of Aristotelia chilensis Stuntz and their pharmacological effects. Current Pharmaceutical Biotechnology 17: 513-523.

Rubilar, M., Jara, C., Poo, Y., Acevedo, F., Gutierrez, C., Sineiro, J., Shene, C. 2011. Extracts of maqui (Aristotelia chilensis) and murta (Ugni molinae Turcz.): sources of antioxidant compounds and $\alpha$-glucosidase $/ \alpha$-amylase inhibitors. Journal of Agricultural and Food Chemistry 59: 1630-1637.

Ruiz, A., Hermosin, I., Mardones, C., Vergara, C., Herlitz, E., Vega, M., Dorau, C., Winterhalter, P., Von Baer, D. 2010. Polyphenols and antioxidant activity of calafate (Berberis microphylla) fruits and other native berries from southern Chile. Journal of Agricultural and Food Chemistry 58: 6081-6089.

Salvande, M., Figueroa, J.A., Armesto, J.J. 2011. Quantity component of the effectiveness of seed dispersal by birds in the temperate rainforest of Chiloé, Chile. Bosque 32(1): $39-45$.

Silveira, F.A.O., Fernandes, G.W. 2006. Effect of light, temperature and scarification on the germination of Mimosa foliolosa (Leguminosae) seeds. Seed Science and Technology 34: 585-592.

Sharifi, M., Pouresmael, M. 2006. Breaking seed dormancy in Bunium persicum by stratification and chemical substances. Asian Journal of Plant Sciences 5(4): 695-699.

Tanaka, J., Kadekaru, T., Ogawa, K., Hitoe, S., Shimoda, H., Hara, H. 2013. Maqui berry (Aristotelia chilensis) and the constituent delphinidin glycoside inhibit photoreceptor cell death induced by visible light. Food Chemistry 139 : 129-137.

Vogel, H., Rasmilic, I., San Martin, J., Doll, U., González, B. 2005. Medicinal Chilean plants, experiences on domestication and cropping of boldo, matico, bailahuén, canelo, peumo and maqui. University of Talca. Talca, Chile. 192 pp.

YANG, Q.H., YE, W.H., YIN, X.J. 2007. Dormancy and germination of Areca triandra seeds. Scientia Horticulturae 113: 107111.

Recibido: 18.08.2016

Aceptado: 21.09.2017 